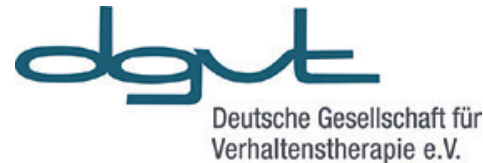

\section{Deutsche Gesellschaft für Verhaltenstherapie (DGVT) e.V.}

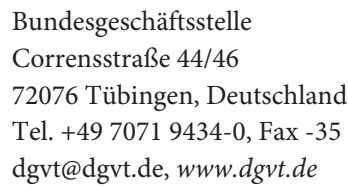

\section{Gute Beratung einfach erkennen - die DGVT und ihr Berufsverband DGVT-BV fördern ein Forschungsprojekt zur Entwicklung von Kompetenzstandards für Berater*innen: Eine bedeutende Erleichterung für Ratsuchende}

Ob es sich um Gesundheits-, Beziehungs-, Erziehungsfragen, um Konflikte im Privatleben oder im Beruf, oder um Entscheidungen bei der Gestaltung des Lebens- und Berufsverlaufes handelt - oft ist bei schwierigen Entscheidungen kompetente, professionelle Beratung angezeigt.

Wie können Ratsuchende unter den unzähligen Berater ${ }^{\star}$ innen, Coachs, Supervisor*innen usw. die Spreu vom Weizen trennen und die tatsächliche Kompetenz eine ${ }^{\star} r$ Berater ${ }^{\star}$ in sicher einschätzen?

Ein Forschungsvorhaben soll Licht ins Dunkel bringen. In den kommenden zwei Jahren wird wissenschaftlich fundiert beschrieben, an welchen überprüfbaren Kompetenzen man gute Berater*innen erkennen kann.

Als einer der in der Deutschen Gesellschaft für Beratung e.V. (DGfB) zusammengeschlossenen 24 Verbände professioneller Berater*innen mit insgesamt über 25.000 Mitgliedern fördern die DGVT und ihr Berufsverband DGVT-BV über 25 Prozent dieses Forschungsprojekts. Das Ergebnis bildet die Grundlage für den „Deutschen Qualifikationsrahmen Beratung" (DQR). Der Qualifikationsrahmen macht Beratungskompetenzen überprüfbar und sichtbar.

Berater*innen, die entsprechende Kompetenzen nachgewiesen haben, können diese dann mit einem europaweit anerkannten Zertifikat belegen. Dadurch erhalten
Ratsuchende und Auftraggeber*innen auf einen Blick Auskunft über geprüfte Beratungskompetenz. Dies verkürzt und erleichtert den Weg zur kompetenten und verlässlichen Beratung entscheidend.

Die Deutsche Gesellschaft für Beratung, unser Dachverband, übernimmt die fachliche und organisatorische Koordination des Projekts. Für die Ausschreibung des Forschungsauftrags ist die Gesellschaft für personzentrierte Psychotherapie und Beratung e.V. (GwG) zuständig. Ein entsprechender Kooperationsvertrag wurde am 18. September 2020 in Köln von den Vorständen der GwG und DGfB unterzeichnet.

Weitere Informationen zur Deutsche Gesellschaft für Beratung (DGfB) und zum Forschungsprojekt sind auf der Website www.dachverband-beratung.de zu finden.

\section{Digitale Hilfe für internationale Krisenregionen - free my mind: Ehrenamtliche psychologische Beratung über das Internet soll Menschen in der ganzen Welt helfen!}

Der größte verhaltenstherapeutische Fachverband in Deutschland, die Deutsche Gesellschaft für Verhaltenstherapie (DGVT) e.V., unterstützt ein Angebot der Firma webPRAX, um Opfern von Krieg, Misshandlung, sozialer Isolation und Unterdrückung schnell und unkompliziert psychologisch zu helfen. Die meisten Menschen, die in internationalen Krisenregionen leben, können von einer medizinischen Versorgung, so wie wir sie in Deutschland genießen, nur träumen. Dies gilt genauso für psychotherapeutische Betreuungsangebote.

Die WHO ermittelte 2019, dass jede fünfte Person in den betroffenen Krisengebieten an einer psychischen Störung leidet, von Angstzuständen über leichte Depressionen bis hin zu Psychosen.

Schlimmer noch, fast jede ${ }^{\star}$ Z Zehnte lebt dort mit einer mittelschweren oder schweren psychischen Störung.

Daher soll ein neuartiges Netzwerk von Psychologischen Psychotherapeut*innen aus westlichen Industrieländern aufgebaut werden, die bereit sind, diesen Menschen über eine Videoplattform ehrenamtlich mit Ihrem Fachwissen und Ihrer Empathie zur Seite zu stehen. Im Rahmen dieses Hilfsprojektes werden befreundete Hilfsorganisationen vor Ort in den Krisenregionen den Kon- 
takt zu den Hilfesuchenden aufnehmen und so eine Verbindung zum neuen Hilfsnetzwerk herstellen. Die Intervention wird dann in einem anonymen Gruppensetting über die webPRAX-Gruppentherapie-Plattform durchgeführt.

Mit nur einer Gruppen-Videosprechstunde pro Woche kann jede ${ }^{\star} r$ Therapeut ${ }^{\star}$ in acht Menschen aus einer Krisenregion gleichzeitig psychotherapeutisch unterstützen.

Es werden in regelmäßigen Abständen Live-InfoWebseminare für interessierte Therapeut ${ }^{*}$ innen angeboten. Eine unverbindliche Anmeldung kann über $w w w$. freemymind.org erfolgen.

Weitere Infos über das Projekt unter

$w w w$.freemymind.org

Weitere Infos über die dgvt:

www.dgvt.de/wir-ueber-uns/

Weitere Infos über webprax unter

www.webprax.de

\section{DGVT-KONGRESS vom 4.-6. März 2021 - ONLINE}

Die Deutsche Gesellschaft für Verhaltenstherapie DGVT e. V. lädt vom 04.-06. März 2021 zum 31. Kongress für Klinische Psychologie, Psychotherapie und Beratung zum Thema „BRAVE NEW PSYCHO THERAPY“ ein. Corona bedingt findet der Kongress online statt, was seiner Vielfalt an Veranstaltungsformen jedoch nicht abträglich ist. In zahlreichen Workshops, Symposien und Diskussionsrunden können sich die Teilnehmer*innen aktiv beteiligen und sich von Diskussionen inspirieren lassen. Der DGVT-Kongress wartet auch 2021 mit einigen Highlights auf Sie. Wir eröffnen den Onlinekongress mit einem Beitrag von Stefan G. Hofmann zum Thema „Process-based therapy“. Weitere Hauptvorträge werden gehalten von: Hanna Christiansen „Kinder von Eltern mit psychischen Erkrankungen", Michael Hengartner „Kritische Auseinandersetzung mit der neurobiogenetischen Depressionsforschung“, Daniela Mier „Funktionelle Bildgebung sozialer Wahrnehmung bei Schizophrenie - Neue Impulse für die Psychotherapie" und Andreas Zick „Rechtsextremismus und gruppenbezogene Menschenfeindlichkeit“.
Ausführliche und aktuelle Informationen zum 31. Kongress für Klinische Psychologie, Psychotherapie und Beratung finden Sie auf unserer Homepage unter $w w w$. dgvt-kongress.de.

\section{Fort- und Weiterbildung}

Die 9. Sommerakademie der DGVT Fort- und Weiterbildung wird vom 03.-05. Juni 2021 in Rostock-Warnemünde zu Gast sein. In vier verschiedenen Workshops werden sich die Teil-nehmer*innen mit dem Thema „Mit den passenden Worten" auseinandersetzen. Ein Vortrag zum Thema „Sprache in der Psychotherapie - Psychotherapie ist Sprachkunst" sowie ein (regeneratives) Beiprogramm runden das Tagungsangebot ab.

In Kooperation mit der Deutschen Gesellschaft für Systemische Therapie, Beratung und Fa-milientherapie (DGSF) und der Systemischen Gesellschaft (SG) wird die DGVT e. V. eine Fachtagung zum Thema „Systemische Therapie meets Verhaltenstherapie - an envolving affair - „Ich sehe was, was du nicht siehst..." vom 15.-16. Oktober 2021 in Erlangen veranstalten. Das Tagungsprogramm setzt sich aus Expert ${ }^{\star}$ innenvorträgen, Live-Arbeit, Kurz-Workshops und einer interaktiven Reflexion zusammen.

Die 19. DGVT-Praxistage der Kinder- und Jugendlichenpsychotherapie mit dem Titel „Von A wie Angst bis Z wie Zeugnis - Schule meets Psychotherapie“ finden vom 27.-28. November 2021 in Magdeburg statt. Im Rahmen von Kurz- und Langworkshops befassen sich Teilnehmer*innen mit Inhalten rund um das Thema Psychotherapie und Schule. Für den Eröffnungsvortrag konnten wir Günter Esser zum Thema „Aktuelle Entwicklungslinien in der verhaltenstherapeutischen Kinder- und Jugendlichenpsychotherapie: Forschungsbefunde und Herausforderungen" gewinnen.

Ausführliche Informationen zu diesen und weiteren Angeboten erhalten Sie unter www.dgvt-fortbildung.de oder über die DGVT-Bundesgeschäftsstelle in Tübingen, Tel.: 070719434 34, Fax: 070719434 35, E-Mail: fortbildung@dgvt.de. Sprechzeiten: Di und Do 9:00-12:00 Uhr; Di und Mi 14:00-15:30 Uhr. 
Deutsche Gesellschaft für Verholtensmedizin und Verhaltensmodifikation e.V.

\section{Deutsche Gesellschaft für Verhaltensmedizin und Verhaltensmodifikation (DGVM)}

\author{
Prof. Dr. Thomas Kubiak \\ Psychologisches Institut \\ Abteilung Gesundheitspsychologie \\ Johannes Gutenberg-Universität Mainz \\ Binger Straße 14-16 \\ 55122 Mainz
}

\section{Aus dem Vorstand}

Angesichts der auch für das kommende Jahr unklaren Auswirkungen und Entwicklungen im Zusammenhang mit der COVID-19 Pandemie wird die DGVM anstelle des etablierten Kongresses eine Veranstaltung im Onlineformat umsetzen, die speziell auf Nachwuchswissenschaftlerinnen und Nachwuchswissenschaftler zugeschnitten ist. Geplant sind folgende Formate

- Ausgewählte Keynote-Vorträge von Expertinnen und Experten zu „hot topics“ der Verhaltensmedizin

- Vorstellung und Diskussion von Promotionsvorhaben von DGVM-Nachwuchswissenschaftlerinnen und Nachwuchswissenschaftlern

- Launch eines Mentoring-Programms, das Nachwuchswissenschaftlerinnen und Nachwuchswissenschaftler erfahrene Mentorinnen und Mentoren aus der DGVM zur Seite stellt

- Workshops zu ausgewählten verhaltensmedizinischen Inhalten und Methodik und Planen der akademischen Karriere.

Die Veranstaltung wird im Oktober 2021 stattfinden. Die Teilnahme ist für Nachwuchswissenschaftlerinnen und Nachwuchswissenschaftler der DGVM kostenfrei.

Wir schreiben erneut unser Peer-Mentoring-Programm für Nachwuchswissenschaftlerinnen und Nachwuchswissenschaftler aus. Wir freuen uns besonders, in der diesjährigen Ausschreibung des erfolgreichen Programms den Umfang der Förderung erhöhen zu können, um den wissenschaftlichen Nachwuchs noch ausdrücklicher unterstützen zu können. Die Details der Ausschreibung finden Sie auf der DGVM-Webseite: $h t t p s: / / w w w$. dgvm-online.de/index.php/nachwuchsfoerderung.

Es gibt zwei Förderlinien:

A. Zuschuss zur Förderung von Promovierenden im Themenbereich der Verhaltensmedizin: Zwei Promovierende finden sich zu einem Peer Mentoring-Team zusammen.

B. Zuschuss zur Förderung von Nachwuchswissenschaftlerinnen und Nachwuchswissenschaftlern nach der
Promotion (PostDocs) im Themenbereich der Verhaltensmedizin: Zwei Nachwuchswissenschaftlerinnen/ Nachwuchswissenschaftler nach der Promotion (PostDocs) oder ein/e PostDoc und ein/e Promovierende/r finden sich zu einem Peer Mentoring-Team zusammen.

Anträge sind als PDF via E-Mail bis zum 31. Dezember 2020 beim 1. Vorsitzenden der DGVM, Prof. Dr. Thomas Kubiak (kubiak@uni-mainz.de), einzureichen.

\section{Berufsrelevante Neuigkeiten}

Bernd Leplow (Halle) fungiert fortan als Strategischer Leiter des „Instituts für Therapie- und Gesundheitsforschung, IFT-Nord“ in Kiel.

Christina Hunger-Schoppe (Witten-Herdecke) ist Mitglied der Leitliniengruppe zur Überarbeitung der S3 Leitlinie „Behandlung von Angststörungen“.

Beate Ditzen (Heidelberg) und Christian Schaaf (Heidelberg) wurde vom Bundesministerium für Gesundheit das Projekt „GenKI - Genetische Beratung zwischen KI und persönlicher Entscheidung" bewilligt, Laufzeit 3 Jahre, Fördersumme 380032 EUR.

Melanie Fischer (Heidelberg) erhielt eine Förderung durch das Margarete von Wrangell-Habilitationsprogramm für Frauen (Ministerium für Wissenschaft, Forschung und Kunst Baden-Württemberg/Europäischer Sozialfonds; Laufzeit 5 Jahre, 350000 EUR) für die Durchführung ihres Vorhabens „Dyadic mechanisms of mental disorders: Behavioral processes, psychobiology, and emotion regulation in the couple context."

\section{Professuren, Preise, Auszeichnungen etc.}

Christina Hunger-Schoppe hat am 1.9.2020 den Lehrstuhl für Klinische Psychologie und Psychotherapie III an der Universität Witten-Herdecke übernommen.

Johannes Laferton ist seit dem 1.10.2020 Professor für Medizinische Psychologie an der Health and Medical University Potsdam.

\section{Diagnostikrelevante Nachrichten (Diagnosekriterien, Messinstrumente, etc.)}

Fragebogen zur Angehörigen-Resilienz und -Belastung (FARBE) - Ein Screeninginstrument für pflegende Angehörige. Ziel des FARBE-Fragebogens ist es, individuelle Resilienz- und Belastungsfaktoren von pflegenden Angehörigen zu identifizieren. Er ist primär für die Anwendung in einem Beratungskontext konzipiert, um einerseits schnell pflegende Angehörige in einer Risiko- 
konstellation zu identifizieren und andererseits basierend auf den individuellen Antworten zu wichtigen Beratungsthemen zu informieren. Der Fragebogen ist in zwei Versionen verfügbar - eine allgemeine Version unabhängig vom Erkrankungsbild des zu Pflegenden und eine spezielle Version für pflegende Angehörige von Menschen mit Demenz. Der Fragebogen besteht aus vier Skalen, von denen zwei Resilienzfaktoren (,Meine innere Haltung', ,Meine Energiequellen') und zwei Belastungsfaktoren (,Schwierigkeiten im Umgang mit der pflegebedürftigen Person', ,Allgemeine Belastungen meiner Lebenssituation') abbilden. Jede Skala weist bei auffälligen Werten spezifische Handlungsempfehlungen für die Beratung und Begleitung pflegender Angehöriger auf. Der Fragebogen ist frei verfügbar und kann zusammen mit dem Manual und den Auswertungstool von folgender Seite heruntergeladen werden: $w w w . z q p$.de/frageboegen-farbe

Wuttke-Linnemann A., Halsband C. A. \& Fellgiebel, A. (2020). Fragebogen zur Angehörigen-Resilienz und -Belastung (FARBE). Berlin: Zentrum für Qualität in der Pflege (ZQP).

\section{Arbeitsgemeinschaft für Verhaltensmodifikation (AVM)}

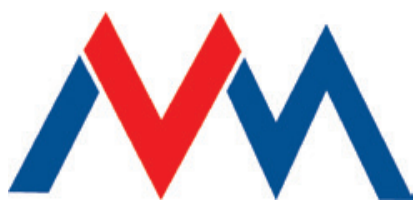

\author{
AVM Österreich \\ Arbeitsgemeinschaft für Verhaltensmodifikation (AVM) \\ Büro Salzburg \\ Paris-Lodron-Straße 32, 5020 Salzburg, Österreich \\ Tel. +43662884166 \\ Büro Wien \\ Leidesdorfgasse 11-13/Stiege 1/Top 8, 1190 Wien, Österreich \\ Tel. +4313204132 \\ office@institut-avm.at, https://institut-avm.at
}

\section{Kompetenzorientierte Supervision in der Verhaltenstherapie}

Die Verhaltenstherapie umfasst ein breites Spektrum an Methoden zur Behandlung von Menschen mit psychischen Erkrankungen. Viele sind hier primär an der Vermittlung von Techniken orientiert, die auf wissenschaftlichen Erkenntnissen beruhen (z. B. Lerntheorien). Die Erweiterung der Verhaltenstherapie in der dritten und vierten Welle bezieht auch Methoden aus ande-

\section{Internationales (ISBM)}

Der 16. Kongress der International Society of Behavioral Medicine wird vom 07.-11.06.2021 online stattfinden. Er steht unter dem Motto „Interdisciplinary Behavioural Medicine: Systems, Networks and Interventions“. Weitere Informationen finden sich hier http://www. icbm-congress.com/.

Die ISBM hat im Jahr 2019 die Taskforce "Definition, Goals and Organization" eingesetzt, um eine aktuelle Definition von ,Behavioral Medicine' und ihrer charakterisierenden Eigenschaften zu erarbeiten sowie die Ziele und Aufgaben und die Organisation der ISBM zu aktualisieren. Die DGVM war besonders aktiv an dieser Taskforce beteiligt (Maria Kleinstäuber, Claas Lahmann, Bernd Leplow, Ricarda Nater-Mewes, Urs Nater). Die Ergebnisse wurden nun veröffentlicht: Dekker et al. (2020). Definition and characteristics of behavioral medicine, and main tasks and goals of ISBM - an international Delphi study. International Journal Behavioral Medicine doi: 10.1007/s12529-020-09928-y

ren Schulen mit ein (z. B. Emotionsfokussierte Therapie; Greenberg 2015). Dadurch ergibt sich die Problematik des „schulenorientierten Zugehens an psychische Probleme mit unterschiedlichen dahinterstehenden Menschenbildern" und deren Integration in andere Modelle. Wichtige Neuentwicklungen haben eine theoretische Basis, die nicht durch die angeblich vier Grundorientierungen (psychodynamisch; verhaltenstherapeutisch; personenzentriert; systemisch) abgedeckt sind. Dies gilt etwa für die Acceptance and Commitment Therapy (ACT; Hayes, Wilson und Strosahl, 2014), aber auch für achtsamkeitsbasierte Therapieansätze (MBST/MBCT; Michalak et al., 2012). Auf diese Problematik weist etwa Rief (2019) hin, der auch auf die Notwendigkeit einer kompetenzorientierten Vermittlung von Psychotherapie an die Ausbildungskandidat ${ }^{\star}$ innen hinweist. Es geht also primär um die Klärung der Frage, was Psychotherapeut ${ }^{*}$ innen können sollen und nicht so sehr darum, inwieweit sie ein theoretisches Schulenkonzept einhalten.

Rief (2019) beschreibt hier folgende Basiskompetenzen für Psychotherapeut*innen:

- Orientierung an einem übergeordneten Störungsmodell (vgl. Rief und Strauß, 2018, S 402)

- persönliche Kompetenzen (z. B. Empathie, soziale Kompetenzen ...)

- Kompetenzen zur Durchführung settingabhängiger Psychotherapie (z.B. Einzeltherapie, Paartherapie, Gruppentherapien, systemische Interventionen) 
- Kompetenzen zur Durchführung störungsabhängiger Psychotherapie entsprechend aktueller wissenschaftlich fundierter Leitlinien (z. B. DBT; Schematherapie; Soziale Phobie vgl. Consbruch et al., 2012)

- Kompetenzen zur personalisierten und komplexitätsabhängigen Psychotherapie (z.B. die Integration kulturspezifischer Aspekte, Alter, Abwägung von Chancen und Risiken der Behandlung ...)

An diesen Kompetenzprofilen müssen sich auch die Ausbildung, Weiterbildung und Supervision orientieren, sodass neue Entwicklungen leicht integriert werden können.

Insofern muss kompetenzorientierte Supervision diese Kriterien berücksichtigen und nicht nur am Vermitteln von „Techniken“ orientiert sein. Dadurch ergeben sich auch neue Aufgaben für Supervisior ${ }^{\star}$ innen (vgl. Zarbock, 2016)

- Die Orientierung an Aufgaben und Kompetenzen in der Supervision

- Die Kompetenz-Trainings-Spirale (KTS) als didaktisches Konzept

- Supervisorische Grundhaltungen

- Supervisionstechniken

- Schwierige Supervisand ${ }^{*}$ innen und schwierige Supervisor*innen

- Krisen und Entwicklungsverläufe von Supervisand ${ }^{*}$ innen

- Schematherapeutische und interaktionelle Ansätze für die Supervision

- Selbsterfahrungstechniken in der Supervision

Zusätzlich sollten auch neue Formen der Supervision vermehrt integriert werden, wie z. B. das Betrachten und Evaluieren von Therapievideos, Selbstbeurteilungsskalen zur Messung der therapeutischen Kompetenzen bei verschiedenen Krankheitsbildern, Skalen für Supervisand ${ }^{*}$ innen und Supervisior*innen zur Beurteilung des Supervisionsprozesses, Rollenspiele, aber auch ein spezifisches Training der Supervisor*innen zur kompetenzorientierten Supervision. Darauf weisen etliche Autor ${ }^{*}$ innen der CBT hin, die vermehrt ein Training von Supervisior ${ }^{*}$ innen und eine Evaluation des Supervisionsprozesses fordern (vgl. Loades und Armstrong, 2016; Murr et al., 2020).

Diesen neuen Entwicklungen versucht auch die AVMÖsterreich in ihrem Curriculum und der damit verbundenen Ausbildung durch kompetenzorientierte, übergreifende Modelle der Vermittlung therapeutischer Fertigkeiten Rechnung zu tragen. Übergeordnet ist diesem Modell der Ausbildung ein Menschenbild (Störungsmodell) des Menschen als biologisches, denkendes, fühlendes, spirituelles und soziales Lebewesen in einer sozialen und kontextuellen Umwelt (Gatterer, 2019). Leben bedeutet also den Einsatz von Kompetenzen zur Bewältigung von „Lebensaufgaben“ und „Krisen“. Insofern muss
Verhaltenstherapie Patient ${ }^{\star}$ innen diese Kompetenzen vermitteln. Dazu ist es aber auch notwendig, dass Ausbildungskandidat*innen in diesen Kompetenzen trainiert und auch ausgebildet werden. Kompetenzorientierte Supervision ist hier ein wesentlicher Beitrag.

Zusammenfassung

In der aktuellen Zeit hat sich die Anzahl von kompetenzbasierten Ansätzen deutlich erhöht. Darauf muss sich auch die Aus-, Fort- und Weiterbildung von Verhaltenstherapeut ${ }^{*}$ innen orientieren, um den Anforderungen der Zukunft zu entsprechen. Deshalb müssen neue Ansätze kompetenzorientiert in die Verhaltenstherapie integriert werden. Dabei rückt nach Rief (2019) das Qualifikationsziel „fit für die optimale Patient*innenversorgung unter praktischer und wissenschaftlicher Perspektive" auch in der Zukunft und mit neuen Herausforderungen mehr in den Vordergrund. Dem muss aber auch die Ausbildung des Lehrpersonals Rechnung tragen und vermehrt kompetenzorientierte Ansätze in die Supervision integrieren.

Univ.-Doz.Dr. Gerald Gatterer, klinischer Psychologe, Gesundheitspsychologe, Psychotherapeut. Stellvertretender Vorsitzender und wissenschaftlicher Leiter der AVM-Österreich; Leiter des Instituts für Alternsforschung der Sigmund Freud Privatuniversität Wien.

Praxisadresse: Gerontopsychologisches Institut, Schlossmühlgasse 22, 2351 Wiener Neudorf, Österreich

\section{Literatur}

Gatterer, G. (2019) Verhaltenstherapie bei depressiven Störungen im Alter. Psychotherapie Forum 23: 103110.

Greenberg, L. S. (2015). Emotion-focused therapy: Coaching clients to work through their feelings. Washington, DC: American Psychological Association.

Hayes, S. C, Wilson, K. G. \& Strosahl, K. D. (2014). Akzeptanz- \& Commitment-Therapie: Achtsamkeitsbasierte Veränderungen in Theorie und Praxis. Paderborn: Junfermann

Michalak, J., Heidenreich, T. \& Williams, J. M. G. (2012). Achtsamkeit (Vol. 48). Göttingen: Hogrefe

Loades, M., \& Armstrong, P. (2016). The challenge of training supervisors to use direct assessments of clinical competence in CBT consistently: A systematic review and exploratory training study. The Cognitive Behaviour Therapist, 9, E27. doi:10.1017/ S1754470X15000288

Murr, S., Nicklas, L., \& Harper, S. (2020). How does supervision aid cognitive behaviour therapy skill development? Perspectives of CBT trainees: A thematic analysis. The Cognitive Behaviour Therapist, 13, E45. doi:10.1017/S1754470X20000422 
Rief, W. (2019) Von der verfahrensorientierten zur kompetenzorientierten Psychotherapie-Qualifikation. Psychotherapeutenjournal. 261-268. https://www.psychotherapeutenjournal.de/ptk/web.nsf/gfx/34214745651 DB85CC12584740025AACC/\%24file/PTJ_2019-3_online.pdf

Rief, W. \& Strauß, B. (2018). Integratives Störungsmodell als Grundlage der Therapieplanung. Psychotherapeut, 63 (5), 401-408.
Zarbock, G. (Hrsg., 2016) Praxisbuch VT-Supervision: Konzepte und Materialien für die aufgaben- und kompetenzorientierte Supervision (AKOS) von Verhaltenstherapie (DVT-Praxis, Band 4). Pabst Science Publishers: Lengrich

Von Consbruch, K., Clark, D., \& Stangier, U. (2012). Assessing Therapeutic Competence in Cognitive Therapy for Social Phobia: Psychometric Properties of the Cognitive Therapy Competence Scale for Social Phobia (CTCS-SP). Behavioural and Cognitive Psychotherapy, 40(2), 149-161. doi:10.1017/S1352465811000622 